\title{
Research on Innovative Technologies for Digital Construction of Integrated Band
}

\author{
Junyi Shao, Yanlei Song and Xuemei Cao \\ Qingdao Technological University, China
}

\begin{abstract}
Integrated band as a type of new upgrade band for equipment has been widely used in ceiling projects, owing to its concise, applicable, and environmental friendly features. However, there are problems in the construction process of integrated band, like large assembly gap, poor positioning, and poor flatness. To address these issues, this paper firstly proposes three innovative technologies for the comprehensive control of the implementation of ceiling project, including the three-dimensional digital typesetting technology of pipeline + integrated band + aluminous gusset plate, multi-directional cross line laser location \& check technology, and overall lifting \& unified adjustment technology. Furthermore, a complete set of construction technology processes has been presented. Finally, the economic and social benefits of these innovative technologies have been proved by the engineering project.
\end{abstract}

\section{Introduction}

Ceiling project is an important part of interior decoration project. With the development of industry science and technology, more and more equipment terminal are used by people, the number of indoor pipeline are increased correspondingly, which greatly affect the beauty of the ceiling project, however, the appearance of integrated band is a good solution to this problem. Integrated band is a type of newly upgraded equipment, which can integrate the functions of fire sprinkler, lighting, speakers, wireless coverage, air conditioning vents (forward, back), manholes and other terminal equipment. Not only can they optimize the top function, but they can freely combination based on needs of user. Compared to the traditional ceiling project, the ceiling project that using integrated band has a series of advantages, such as simple, practical, saving money, saving time and effort, saving green energy, environmental protection, beautiful and so on. The contrast between traditional ceiling project and integrated band ceiling project in Figure1.

However, for the ceiling project of large space, due to the restriction on the processing modulus of integrated band and the restriction on high-altitude construction, the traditional construction methods mainly exist large assembly gap, poor positioning, poor flatness and other quality problems. Therefore, a comprehensive analysis of co-ordination arrangements and technological innovation should be taken into account, which will greatly accelerate the further use and development of integrated band.
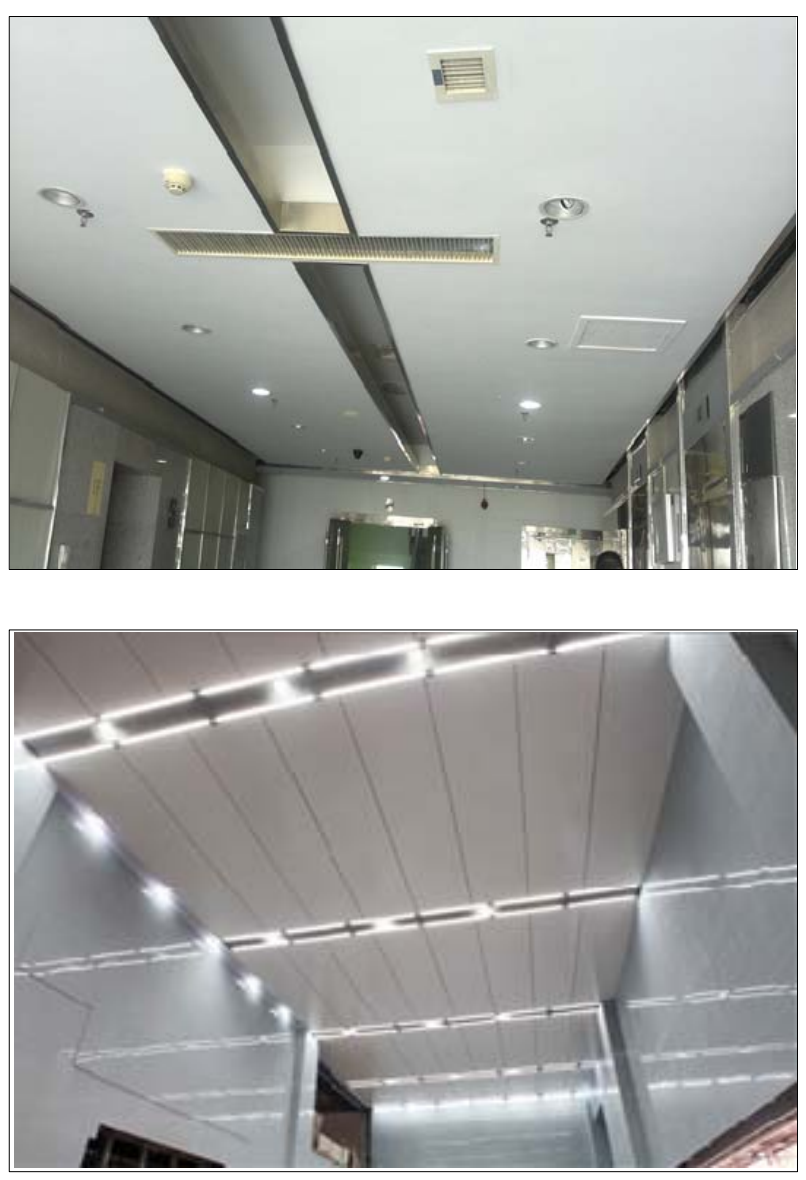

Figure 1. The contrast between traditional ceiling project and integrated band ceiling project.

\section{The key technology integration of integrated band}

\subsection{The three-dimensional digital typesetting technologies of pipeline + integrated band + aluminous gusset plate}

According to the position of integrated band, this paper has presented to establish space model by computer-aided 
design, to reasonably determining the direction and layout of pipeline. Furthermore, combined with the overall design effect, this paper has also presented to calculate the amount and size of aluminous gusset plate scientifically and accurately by using computer software, which can control of the installation of aluminous gusset plate effectively. These technologies not only improve the construction efficiency, but also achieve the twin goals of aesthetics and cost savings. Detail in Figure2.

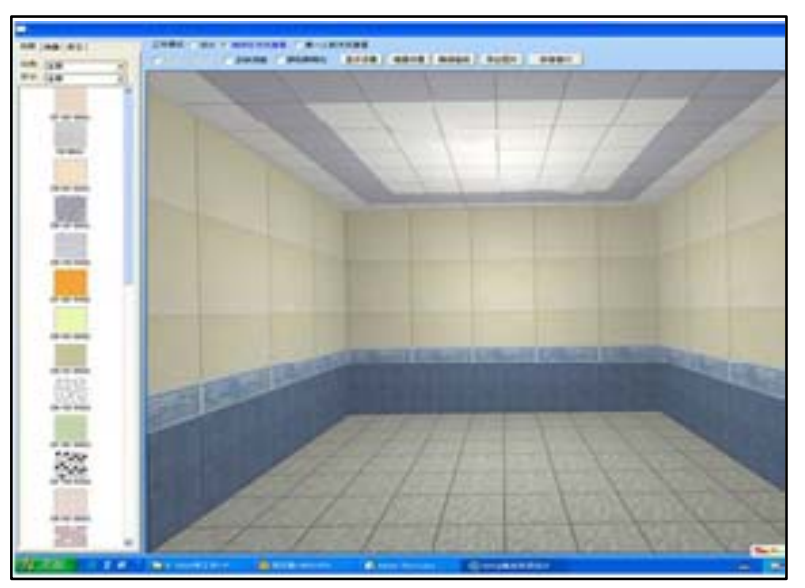

Figure2. Three-dimensional digital typesetting.

\subsection{Multi-directional cross line laser location and check technologies}

Aiming at the problem of poor positioning during construction, this paper has presented to use cross line laser ${ }^{[1]}$, which can achieve the function of accurate positioning by making full use of the horizontal lines and vertical lines projected by the instrument. According to the known standard point, this paper has presented to establish a system of axis control network through unified measurement. Then integrated band, keel, hanging points and other locations will be implemented the process of lofting in accordance with the proportion of 1:1. In order to control the accuracy of the discharge line, the process of lofting must be strict and careful. These technologies can improve the installation accuracy of the integrated band. Meanwhile, they can speed up the construction schedule. Detail in Figure3.

\subsection{Overall lifting and unified adjustment technologies}

Due to the problem of poor flatness and the difficulties in the process of integrated band high-altitude assembly operations, this paper has presented to overall lifting and unified adjustment technologies to solve these problems. According to the design drawings, completing the assembling of integrated band shell in the ground, and then adjusting the gap of integrated band shell by node fastener. After the gap adjustment is completed, this paper has presented to use the lifting platform to implement the overall lift of integrated band shell, then to implementing disposable and unified adjustment of integrated band shell's flatness by using the cross line laser and laser level, to ensure that the integrated band high-quality installation, smooth and beautiful.

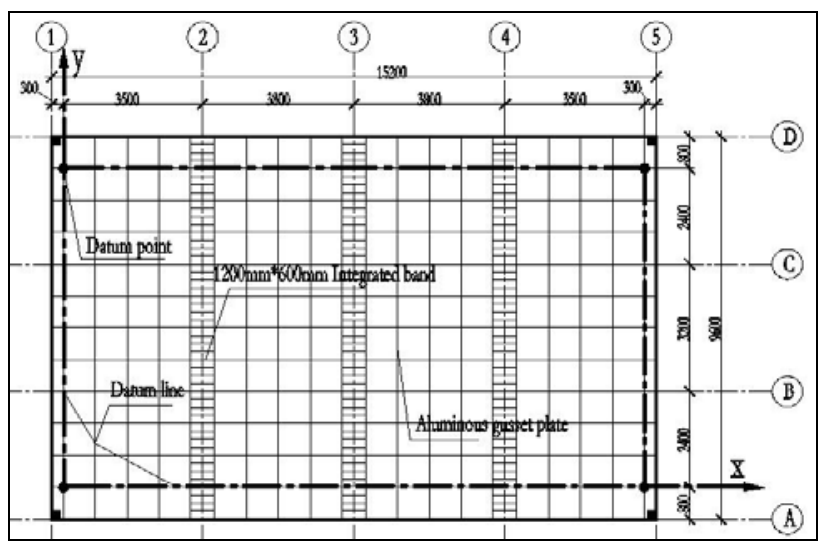

Figure3. The system of axis control network.

\section{Construction technological process of innovative technologies}

In order to ensure the smooth implementation of the innovative technologies, this paper presents a complete set of construction technology processes. Detail in Figure4.

\section{The main technical operation points of innovative technologies}

\subsection{Deepening the design drawings}

1) According to design position of integrated band, considering the position of air conditioner reserved holes, spraying reserved holes and other terminal device pipeline reserved holes in the integrated band shell, establishing 3D model by using computer-aided design. Arranging the wind pipe, water supply and drainage, fire and other pipeline uphold the principle of convenient installation and maintenance. Take the following measurements on the premise that the structure and ceiling elevation can't be affected firstly. Pipe with pressure avoids pipe without pressure, small pipe avoid large pipe, water pipe avoid wind pipe.

According to the results of the $3 \mathrm{D}$ simulations, operating personnel analysis and determine which pipelines are in conflict and need to deal with. If there are conflicts of pipelines, they should be removed in accordance with the principle of less turning. 


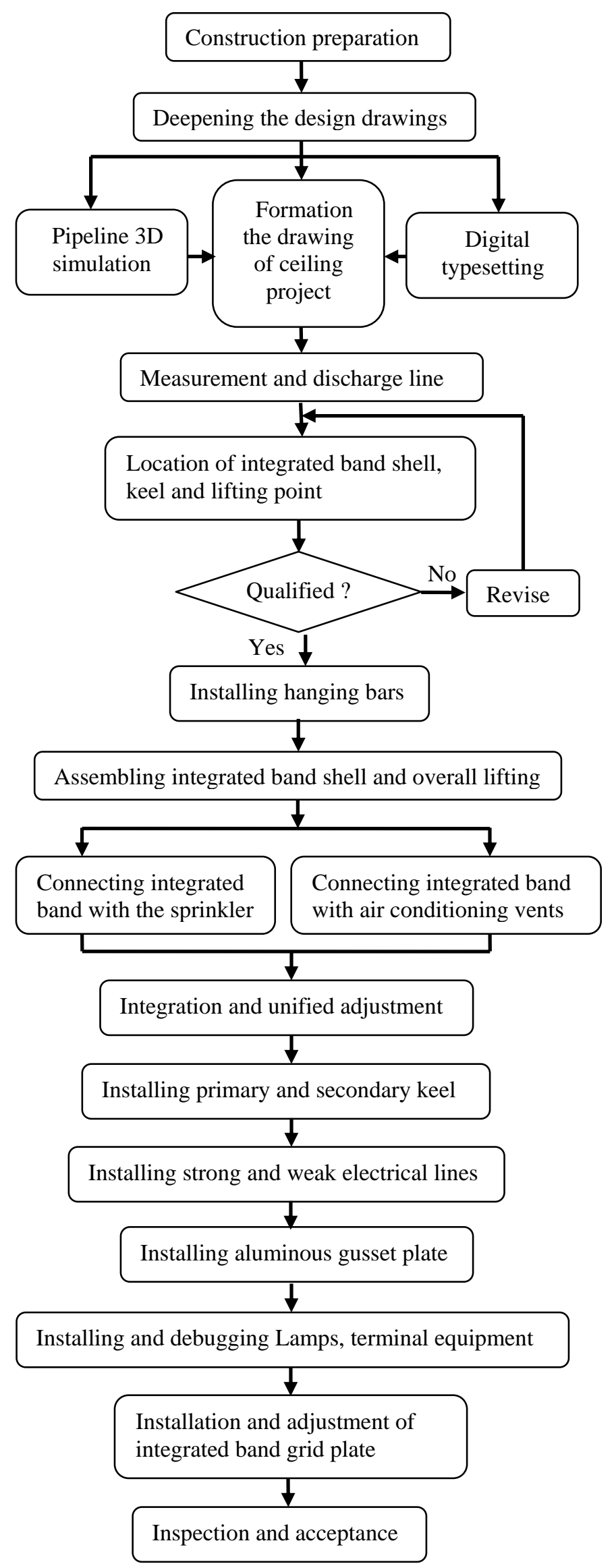

Figure4. The construction flowchart of innovative technologies.

2) On the basis of integrated band size and location, determining the optimal layout and cut size of aluminous gusset plate by using computer digital typesetting technologies.

\subsection{Measurement and discharge line}

1) According to the reference point drawings, selecting a point as the benchmark control point, finding out other control point on the ground at the method of comprehensive measurement and centralized control, linking the control points to form a global control network.

2) Checking the control point after the completion of the measurement. The control point elevation error should not exceed $\pm 1 \mathrm{~mm}$. At the same time, operators need to fill in the records of axis and control line.

3) According to the design drawings, lofting the position of integrated band, keel and hanging points in accordance with the proportion of 1:1 on the ground.

4) Copy measuring level point at each wall (column) angle (if the wall long, it should be appropriate to increase the copy measuring points at middle location) by using laser level, linking the level points to form level lines. The height of these level lines from the ground is + $500 \mathrm{~mm}$. According to the design height of ceiling, checking and controlling the height of the ceiling by using of the cross line laser, and the maximum elevation error should not exceed 1mm. Detail in Figure5.

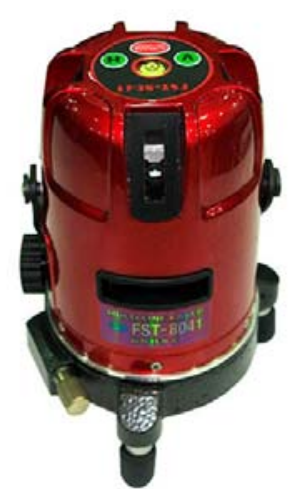

Figure5. The schematic diagram of cross line laser.

\subsection{Hanger rod installation}

1) The installation of hanger rod adopts expansion bolt and anchor bolt methods. The model of hanger rod is Round Steel, whose diameter is $8 \mathrm{~mm}$. The spacing between the hanger rods is $1000 \mathrm{~mm}$.

2) According to drawings, leaving the cross line laser on the reference point of axis control network, removing the cross line laser, ensuring all lifting points of the main keel and integrated band are projected to the ceiling. The intersection of the vertical and horizontal laser line is the position of hole.

3) Using hammer with anti-noise and dust transparent fiberglass cover to implement the process of drilling, after the completion of drilling, fixing the hanger rod with expansion bolts. After the completion of the whole, using cross line laser implement overall review. 


\subsection{Integrated band shell installation and overall lifting}

1) In accordance with design drawings, completing the assembling of integrated band shell in the ground, and then adjusting the gap of integrated band shell by node fastener, and the maximum gap between the integrated band shells should not exceed $0.5 \mathrm{~mm}$.

2) Using mobile hydraulic lifting platform to lift the integrated band shells that has assembled. Making sure the lifting platform is in good working condition before lifting. At the process of lifting, strengthen the protection of the connecting part of integrated band shell. Detail in Figure6.

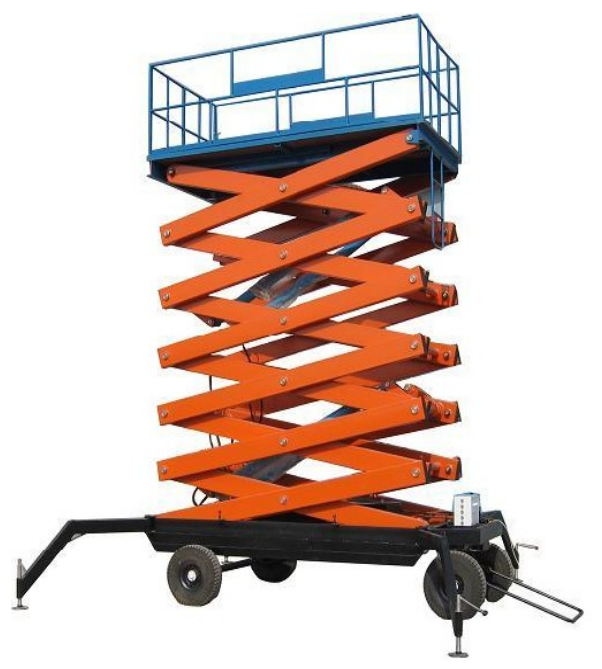

Figure6. The schematic diagram of lifting platform.

\subsection{Primary and secondary keel of aluminous gusset plate installation}

1) The model of primary keel ${ }^{[2]}$ adopt C38 light steel keel and the spacing between the primary keels is $1200 \mathrm{~mm}$.

2) According to the specification of aluminous gusset plate size, selecting the matching model of secondary keel. The secondary keels are hanging in the main keels through hanger.

\subsection{Aluminous gusset plate installation}

1) Inspecting the performance of aluminous gusset plate, which including size, color and specifications. ensuring the quality of materials meet the requirements.

2) Pressing both sides of aluminous gusset plate gently and inserting the flange portion into keel. After the installation is completed, using cross line laser to review the flatness of aluminous gusset plate.

\subsection{Completion and acceptance}

In the process of project completion and acceptance, the following documents and records shall be checked: construction drawings of ceiling project, the layout drawings of integrated band, the design description and other design documents, the product qualification certificate, the performance test report, the inspection report and so on. If the above documents are available, checking the construction quality of the site whether meets the contract requirements.

\section{Application examples}

The integrated band innovative construction technology has been applied to a project of Qingdao Development Zone. The project is an office building with refined decoration and total construction area of the office building is 34832.32 square meters, the ceiling project adopt the integrated band.

It has been proven through practice that the proposed integrated band digital construction technologies can achieve twin objectives of saving and aesthetics. The technologies can ensure the accuracy of integrated band and pipeline position, save the amount of aluminous gusset plate and improve installation efficiency of keel and integrated band. Not only can these technologies reduce the cost of quality, but also they can improve the social benefits.

\subsection{Economic benefit analysis}

Because of the construction technology integration and change, speeding up the construction process and saving construction cost. A total saving of 168,200 RMB construction costs, economic benefits is remarkable.

\subsection{Social benefit analysis}

1) Convenience performance: pipeline \& integrated band \& aluminous gusset plate $3 \mathrm{D}$ digital simulation systems guarantee the accuracy of the component position, ensuring the subsequent process of installation convenient and quick.

2) Aesthetics performance: multi-directional cross line laser location and check technologies avoid the generation of installation gap effectively, which ensure the smoothness and aesthetics of ceiling project.

3) environmental protection performance: First, the integrated band is an environmentally friendly material, in addition, the use of new technologies can reduce the cut of aluminous gusset plate, which reducing the amount of dust and noise.

The key technologies of this paper proposed are mature and reliable. The process of construction is environmental friendly and energy-saving, which winning the high user satisfaction. Social benefits is remarkable

\section{References}

[1] Yi-song Zhou, Qing-chao Hu, The application of cross line laser in the manufacture of steel welding, Heavy Industrial \& Hoisting Machinery. 3(2014):2728.

[2] Chang-gui Wang, The aluminum gusset plate ceiling construction process of large-area gymnasium, Anhui Architecture. 5(2001):32-33. 